\title{
Distinct Cerebellar Contributions to Intrinsic Connectivity Networks
}

\author{
Christophe Habas, ${ }^{1 *}$ Nirav Kamdar, ${ }^{2 *}$ Daniel Nguyen, ${ }^{2}$ Katherine Prater, ${ }^{2}$ Christian F. Beckmann, ${ }^{3}$ Vinod Menon,,${ }^{2,4}$ and \\ Michael D. Greicius ${ }^{2,4,5}$ \\ ${ }^{1}$ Service de NeuroImagerie, Hôpital des Quinze-Vingts, Université Pierre et Marie Curie Paris 6, 75012 Paris, France, ${ }^{2}$ Department of Psychiatry and \\ Behavioral Sciences, Stanford University School of Medicine, Palo Alto, California 94304, ${ }^{3}$ Oxford Centre for Functional Magnetic Resonance Imaging of the

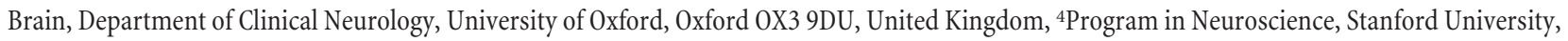 \\ Stanford, California 94305-5173, and ${ }^{5}$ Functional Imaging in Neuropsychiatric Disorders Laboratory, Department of Neurology and Neurological Sciences, \\ Stanford University School of Medicine, Stanford, California 94305-5235
}

Convergent data from various scientific approaches strongly implicate cerebellar systems in nonmotor functions. The functional anatomy of these systems has been pieced together from disparate sources, such as animal studies, lesion studies in humans, and structural and functional imaging studies in humans. To better define this distinct functional anatomy, in the current study we delineate the role of the cerebellum in several nonmotor systems simultaneously and in the same subjects using resting state functional connectivity MRI. Independent component analysis was applied to resting state data from two independent datasets to identify common cerebellar contributions to several previously identified intrinsic connectivity networks (ICNs) involved in executive control, episodic memory/selfreflection, salience detection, and sensorimotor function. We found distinct cerebellar contributions to each of these ICNs. The neocerebellum participates in (1) the right and left executive control networks (especially crus I and II), (2) the salience network (lobule VI), and (3) the default-mode network (lobule IX). Little to no overlap was detected between these cerebellar regions and the sensorimotor cerebellum (lobules V-VI). Clusters were also located in pontine and dentate nuclei, prominent points of convergence for cerebellar input and output, respectively. The results suggest that the most phylogenetically recent part of the cerebellum, particularly crus I and II, make contributions to parallel cortico-cerebellar loops involved in executive control, salience detection, and episodic memory/self-reflection. The largest portions of the neocerebellum take part in the executive control network implicated in higher cognitive functions such as working memory.

\section{Introduction}

Resting-state functional connectivity studies represent a rapidly growing subfield of human brain mapping. Biswal et al. (1995) were the first to demonstrate the potential of this approach, showing that low-frequency fluctuations of the blood-oxygen level-dependent (BOLD) signal of the left motor cortex were temporally correlated with fluctuations in the right motor cortex and the bilateral somatosensory cortex. Subsequently, restingstate functional MRI (fMRI) studies have replicated this sensorimotor network (Xiong et al., 1999; Beckmann et al., 2005) and demonstrated additional intrinsic connectivity networks (ICNs) corresponding to basic functions such as vision, audition, language, episodic memory, executive function, and salience detection (Cordes et al., 2000; Greicius et al., 2003; Kiviniemi et al., 2003; Fox et al., 2005; Fransson, 2005; Seeley et al., 2007). Multi-

Received April 20, 2009; revised May 18, 2009; accepted May 20, 2009.

This work was supported by National Institutes of Health Grants NS048302, HD047520, and NS058899, and National Science Foundation Grant BCS/DRL 0750340

${ }^{*}$ C.H. and N.K. contributed equally to this work.

Correspondence should be addressed to Dr. Christophe Habas, Service de Neurolmagerie, Hôpital des QuinzeVingts, Université Pierre et Marie Curie Paris 6, 28, rue de Charenton, 75012 Paris, France. E-mail: chabas@quinze-vingts.fr.

DOI:10.1523/JNEUROSCI.1868-09.2009

Copyright $\odot 2009$ Society for Neuroscience $\quad$ 0270-6474/09/298586-09\$15.00/0 modal imaging has demonstrated that functional connectivity in these ICNs has electrophysiological correlates (Laufs et al., 2003) and reflects underlying structural connectivity in humans (Greicius et al., 2009) and nonhuman primates (Vincent et al., 2007). Functions have been attributed to the various ICNs based on their resemblance to networks activated by specific tasks, their relationship to specific cognitive disorders, and their correlation with cognitive and affective measures acquired outside the scanner (Greicius et al., 2004; Fox et al., 2005; Seeley et al., 2007). The focus in these studies has been on cortical contributions to ICNs. Some efforts have been made to examine subcortical contributions, but these have generally been limited to the basal ganglia, thalamus, and hypothalamus with scant attention to cerebellar contributions. Only two previous studies have explored restingstate cerebellar connectivity. He et al. (2004) and Allen et al. (2005) used a limited region-of-interest (ROI) approach to demonstrate cerebellar connectivity with the basal ganglia, thalamus, prefrontal and parietal cortices.

The general lack of attention to the cerebellum in ICNs is in keeping with a long-standing tendency to underplay cerebellar contributions to nonmotor functions. This is despite the fact that a staggering amount of convergent, multimodal data has been gathered to build a strong case for the role of the cerebellum in various cognitive and affective functions. Several studies have 
substantiated prominent connections from associative and limbic cortices and hypothalamus to the neocerebellum (including the dentate nuclei) (Schmahmann, 1996; Haines et al., 1997; Middleton and Strick, 1997, 2001; Dum and Strick, 2003). These cerebral afferents quantitatively increase from apes to human (Ramnani et al., 2006) and mainly reach the most phylogenetically recent parts of the cerebellum: lobules VI-VII (Kelly and Strick, 2003). A recent meta-analysis of neuroimaging studies of the neocerebellum has documented its role in emotion, language, working memory, and executive functions (Stoodley and Schmahmann, 2009). Furthermore, Schmahmann and Sherman (1998) followed by Levisohn et al. (2000) have described a variety of cognitive and affective impairments in patients suffering from focal cerebellar lesions. These impairments, broadly referred to as dysmetria of thought and emotion, were mainly observed in lesions of lobules VI-VII. Cognitive deficits in executive functions, memory, and spatial cognition occurred with hemispheric lesions, whereas affective disturbances occurred with vermian lesions. Therefore, converging data strongly suggest that the human neocerebellum contributes to parallel associative cerebrocerebellar networks involved in various aspects of cognition and emotion (Schmahmann, 2004). Despite this burgeoning evidence, cerebellar involvement in cognition is still a matter of some debate. For instance, some studies have failed to find significant cognitive impairment in cerebellar lesion patients and in those that have the deficits are often minor and potentially confounded by motor or oculomotor task demands (Helmuth et al., 1997; Thier et al., 1999; Haarmeier and Thier, 2007).

In the current study, we sought explicitly to explore the role of the cerebellum in several previously defined ICNs. We hypothesized that the sensorimotor ICN would incorporate clusters in the motor regions of the cerebellum, whereas the several ICNs linked to cognitive or affective processing would incorporate distinct neocerebellar clusters.

\section{Materials and Methods Overview}

To test our hypothesis, we first applied independent component analysis (ICA) to the resting-state fMRI data of 15 healthy control subjects. Using an unbiased template-matching procedure (Greicius et al., 2004), we identified the following ICNs: the default-mode network (DMN) (Greicius et al., 2003, 2004), the executive control network (ECN; divided by ICA into left and right hemisphere ECNs) (Seeley et al., 2007), the salience network (Seeley et al., 2007), and the sensorimotor network (Biswal et al., 1995; Xiong et al., 1999). The same five ICNs were then identified in a second dataset to examine, in detail, the replicable cerebellar connectivity in each ICN. Subjects in both datasets also underwent structural scans and various task-activation fMRI scans which were not used in this study.

\section{First (Paris) dataset \\ Subjects}

Fifteen healthy subjects (ages, 19-40; mean age, 26.5; nine females, all right-handed) were scanned after giving written informed consent.

\section{MRI data acquisition}

Functional images were acquired on a whole-body 3T scanner (Signa Horizon; GE Healthcare), using an eight-channel head coil. In each scanning sequence, 32 contiguous axial T2*-weighted gradient echo-planar images [echo time (TE), $40 \mathrm{~ms}$; repetition time (TR), $2500 \mathrm{~ms}$; field of view, $30 \times 30$; matrix, $128 \times 128 \mathrm{~mm}$, zero filled to $256 \times 256 \mathrm{~mm}$; slice thickness, $4 \mathrm{~mm}$; interslice gap, $0 \mathrm{~mm}$ ), were obtained to encompass the entire brain, brainstem, and cerebellum. Two hundred and sixteen volumes were acquired for the resting-state functional scan, with four "dummy" volumes acquired at the start of the session to allow for steadystate magnetization.

For the resting-state scan, subjects were instructed to keep their eyes closed and try to hold still. The scan lasted $9 \mathrm{~min}$ and $10 \mathrm{~s}$.

\section{Preprocessing}

Functional MRI data were format converted from dicom to analyze format using MRIcro (http://www.sph.sc.edu/comd/rorden/mricro.html) and then analyzed using SPM5 analysis software (http://www.fil.ion.ucl.ac.uk/spm). Images were realigned to correct for motion, corrected for errors in slice timing, spatially transformed to standard stereotaxic space [based on the Montreal Neurologic Institute (MNI) coordinate system], resampled to 2 $\mathrm{mm}$ isotropic voxel size using sinc interpolation, and smoothed with a $4 \mathrm{~mm}$ full-width half-maximum Gaussian kernel to decrease spatial noise before statistical analysis. Translational movement in millimeters $(x, y, z)$ and rotational motion in degrees (pitch, roll, yaw) was calculated based on the SPM5 parameters for motion correction of the functional images in each subject. No participants had a range of movement $>3 \mathrm{~mm}$ translation or $3^{\circ}$ of rotation.

\section{Analysis}

For each analysis, an additional four frames of the functional data were discarded to allow for magnet stabilization.

Group-level-independent component analysis. The data were temporally filtered using a high-pass filter of $100 \mathrm{~s}$ and a low-pass filter of $2.8 \mathrm{~s}$. These subject-specific time series data were then concatenated to form a single, group-level four-dimensional dataset from all 15 subjects. This group dataset was then decomposed into independent component maps using a spatial ICA implemented in the MELODIC software (Beckmann and Smith, 2004), part of FMRIB Software Library (FSL) (http:// www.fmrib.ox.ac.uk/fsl). For computational reasons, the number of components was fixed at 30 for the group-level ICA. This initial group ICA decomposition allowed for the generation of group-level templates for the ICNs, which were used to select subject-specific ICA maps obtained from the single-subject analyses described below.

Single-subject-independent component analysis. Spatial ICA was also performed at the single-subject level using the same temporal filtering parameters. At the single-subject level, MELODIC's automated dimensionality estimate was used to select the optimum number of components for each subject. The number of components per subject ranged from 35 to 48 .

Details of the ICA approaches used here are available in earlier publications from the FSL group (Beckmann and Smith, 2004; Beckmann et al., 2005).

\section{Second (Stanford) dataset \\ Subjects}

Twenty-two healthy subjects (ages, 19-21; mean age, 20.6; 11 females, all right-handed) were scanned after giving written informed consent.

\section{MRI data acquisition}

Images were acquired on a 3T GE Signa scanner using a custom-built head coil. Head movement was minimized during scanning by a comfortable custom-built restraint. A total of 29 axial slices $(4.0 \mathrm{~mm}$ thickness) parallel to the anterior commissure-posterior commissure line and covering the whole brain were imaged using a $\mathrm{T} 2{ }^{\star}$-weighted gradient echo spiral pulse sequence (TR, $2000 \mathrm{~ms}$; TE, $30 \mathrm{~ms}$; flip angle, $80^{\circ}$; 1 interleave) (Glover and Lai, 1998). The field of view was $20 \mathrm{~cm}$, and the matrix size was $64 \times 64$, providing an in-plane spatial resolution of 3.125 $\mathrm{mm}$. To reduce blurring and signal loss arising from field inhomogeneities, an automated high-order shimming method based on spiral acquisitions was used before acquiring functional MRI scans (Kim et al., 2002).

For the resting-state scan, subjects were instructed to keep their eyes closed and try to hold still. The scan lasted $8 \mathrm{~min}$.

\section{Preprocessing}

A linear shim correction was applied separately for each slice during reconstruction using a magnetic field map acquired automatically by the pulse sequence at the beginning of the scan. Functional MRI data were then analyzed using SPM5 analysis software (http:// 
www.fil.ion.ucl.ac.uk/spm). Images were realigned to correct for motion, corrected for errors in slice timing, spatially transformed to standard stereotaxic space (based on the MNI coordinate system), resampled to $2 \mathrm{~mm}$ isotropic voxels using sinc interpolation, and smoothed with a $4 \mathrm{~mm}$ full-width halfmaximum Gaussian kernel to decrease spatial noise before statistical analysis. Translational movement in millimeters $(x, y, z)$ and rotational motion in degrees (pitch, roll, yaw) was calculated based on the SPM5 parameters for motion correction of the functional images in each subject. No participants had a range of movement $>3 \mathrm{~mm}$ translation or $3^{\circ}$ of rotation.

\section{Analysis}

For the Stanford data, the first five frames of the functional data were discarded to account for magnet stabilization.

Spatial ICA was again performed at the single-subject level using the same methods described for the Paris dataset. The only difference is that the Stanford data were not temporally filtered before applying ICA. The need for temporal filtering in resting-state ICA studies remains unclear. Here, we have applied it in the first dataset and not in the second. As such, our intersection maps show common cerebellar clusters independent of whether or not temporal filtering was applied before ICA. The number of components in the Stanford data ranged from 25 to 128.

\section{Group results}

Network identification

Identification of resting-state brain networks for each subject was done in three distinct steps: (1) visual identification of five ICNs from the group-level components in the Paris dataset; (2) creating binary masks of each ICN; (3) using the mask as a template to select each individual subject's best-fit component using a template-matching procedure as we and others have done previously (Greicius et al., 2007).

Previously described ICNs corresponding to the sensorimotor network, the DMN (Greicius et al., 2003), the ECN (Seeley et al., 2007), and the salience network were visually identified from the 30 group-levelindependent components (Greicius et al., 2003; Damoiseaux et al., 2006; Seeley et al., 2007). As has been shown previously, the ECN was divided by ICA into homologous left and right networks, referred to here as LECN and RECN, respectively (Damoiseaux et al., 2006). The five ICNs here were selected based, visually, on their correspondence to five ICNs identified by Damoiseaux et al. (2006). Specifically, our DMN corresponds to their Figure $3 B$, our LECN to their Figure 3C, our RECN to their Figure 3D, our salience network to their Figure 3J, and our sensorimotor network to their Figure $3 H$. We confirmed this match by calculating a spatial correlation between each of our five visually selected templates and the eight ICNs shown in Damoiseaux et al. (2006), their Figure 3 (these maps were provided to us by Dr. Damoiseaux, Stanford University School of Medicine, Stanford, CA). In each case, our visually selected template was more strongly correlated with Damoiseaux et al. (2006), their Figure 3 ICN, described above, than with any of the other seven ICNs (i.e., our visually selected DMN was more strongly correlated with their Fig. $3 B$ than with any of the seven other ICNs shown in their Fig. 3). These group-level ICA maps were then binarized to serve as templates for the template-matching algorithm, used to select the best-fit component for each ICN from each subject's individual ICA data. The templatematching algorithm derives a goodness-of-fit score for each of a given subject's several components. The goodness-of-fit is calculated as the mean $z$-score of voxels within the template minus the mean $z$-score of voxels out- side the template. That component with the highest goodness-of-fit score to the template is selected as the best-fit component. After running the template-matching algorithm, separately for each of the five ICN templates, every subject had five best-fit components, one for each ICN.

For each ICN and separately for each of the two datasets, group maps were calculated in SPM 5 by means of a between-subjects random-effects analysis (i.e., a one-sample $t$ test on the best-fit independent components for each subject). We only report and display findings replicated in the two datasets. This was done by generating an intersection map for each ICN limited to voxels that were present in both groups at a $p<0.01$ significance threshold [joint probability (Poline et al.,1997), height and extent, corrected at the whole-brain level, minimum cluster size 25 voxels]. This step ensures that any reported clusters cannot be attributed to site-specific data acquisition or processing steps.

For display purposes, all statistical maps are overlayed on a T1weighted MNI template using MRIcro. Cerebellar cluster localization was determined: by visual inspection using the MRI atlas of the human cerebellum, provided by Schmahmann et al. (2000), and with the probabilistic atlas of the cerebellum (http://www.bangor.ac.uk/ $\sim$ pss412/imaging/propatlas.htm) using the FSL view option of FSL 4.1 (FMRIB Software Library: http://www.fmrib.ox.ac.uk/fsl/).

\section{Distinctness of the cerebellar contributions}

To demonstrate both qualitatively and quantitatively that the cerebellum makes relatively distinct contributions to the five ICNs studied here, we undertook two additional analyses. First, we produced a union map showing the cerebellar contributions from all five ICNs on a single set of cerebellar slices. Second, we generated intersection maps for each possible pairing of 

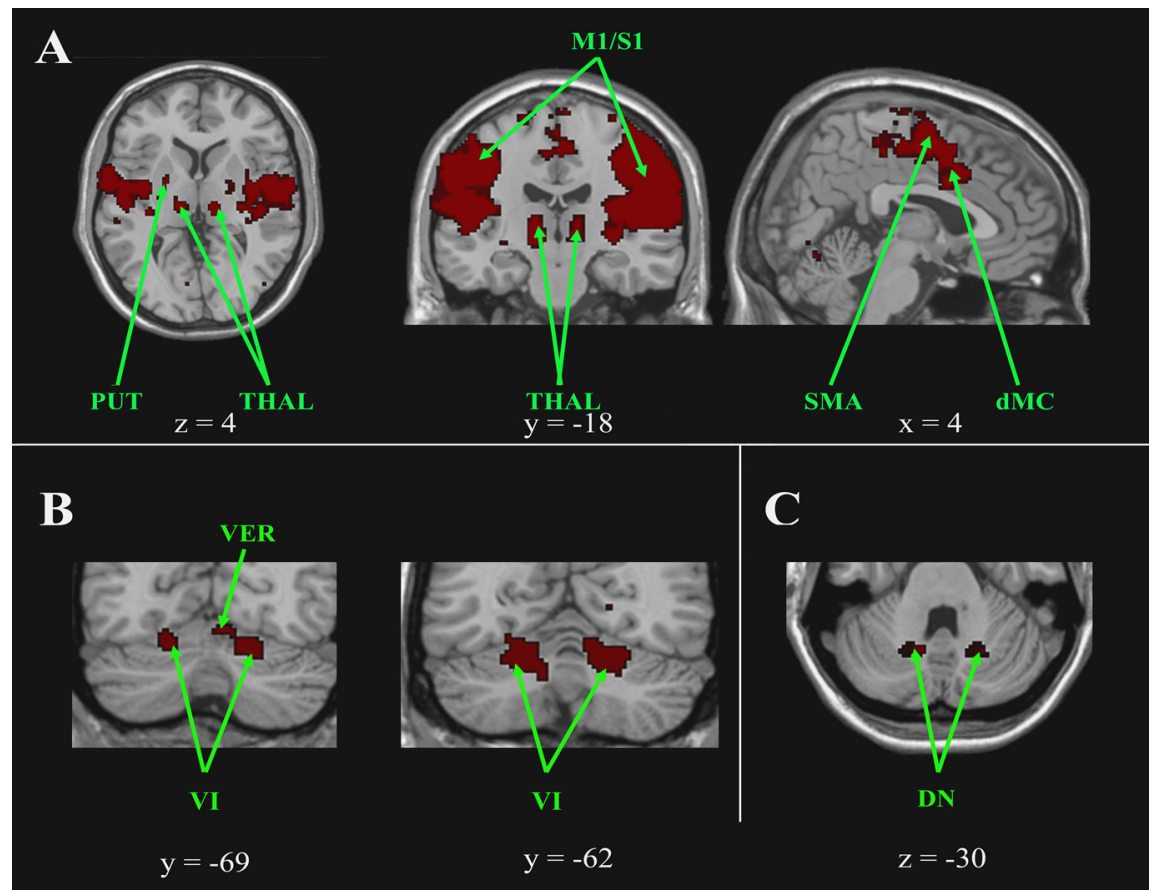

Figure 2. Cortical, subcortical, and cerebellar regions of the sensorimotor network. $A$, Cortical and subcortical regions of the sensorimotor network are shown on axial, coronal, and sagittal slices. $\boldsymbol{B}, \boldsymbol{C}$, Cerebellar regions are shown on coronal slices $(\boldsymbol{B})$ and on an axial slice (C). The left side of the image corresponds to the right side of the brain (radiologic convention). This is an intersection map showing only voxels that were present in the sensorimotor network of both datasets at a corrected threshold of $p<0.01$. CN, Caudate nucleus; dMC, dorsal motormotor cingulate cortex; DN, dentate nucleus; PUT, putamen; RN, red nucleus; SMA, supplementary motor cortex; THAL, thalamus.

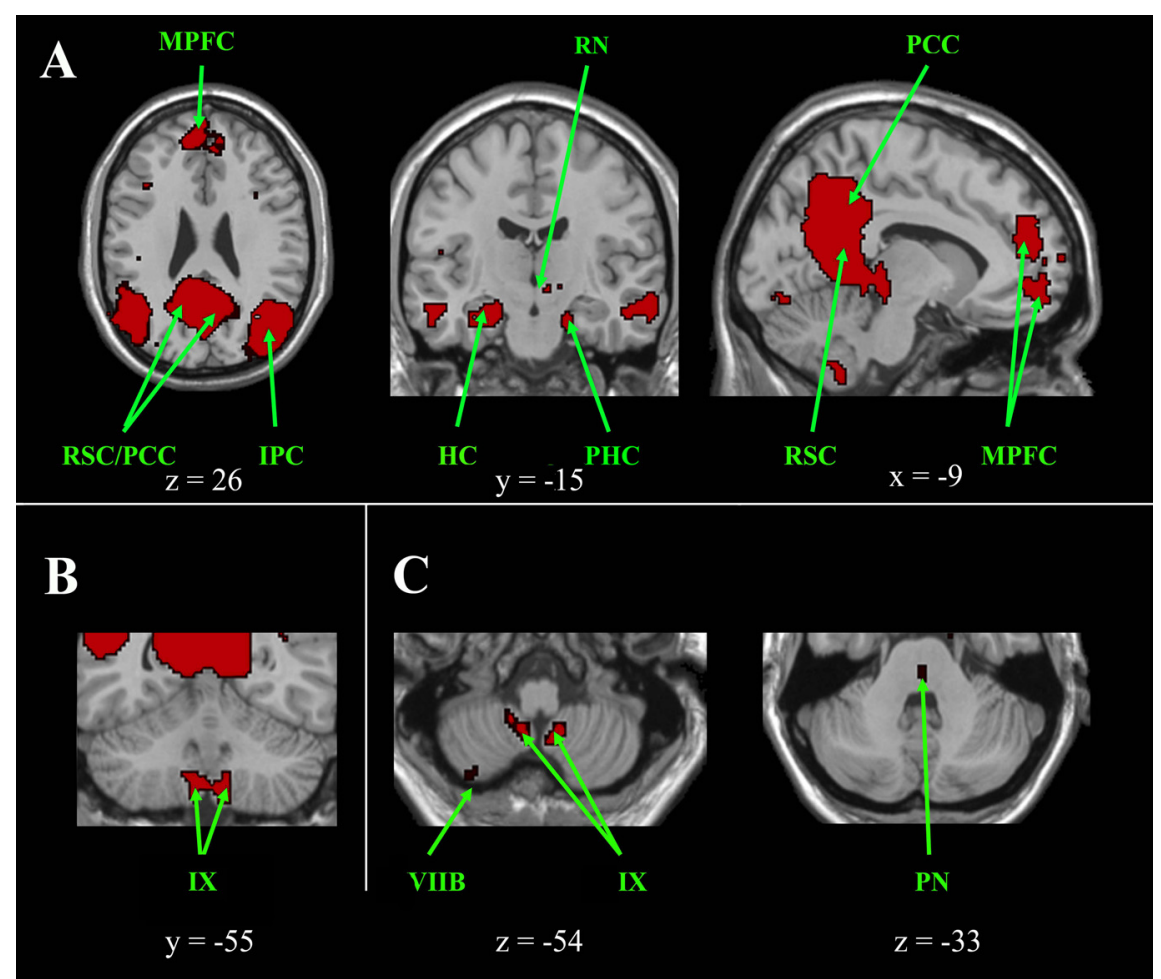

Figure 3. Cortical, subcortical, and cerebellar regions of the default mode network. $A$, Cortical and subcortical regions of the default mode network are shown on axial, coronal, and sagittal slices. $\boldsymbol{B}, \boldsymbol{C}$, Cerebellar regions are shown on a coronal slice $(\boldsymbol{B})$ and on axial slices $(\boldsymbol{C})$, which also highlight a pontine region. The left side of the image corresponds to the right side of the brain. This is an intersection map showing only voxels that were present in the default mode network of both datasets at a corrected threshold of $p<0.01$. HC, Hippocampus; IPC, inferior parietal cortex; MPFC, median prefrontal cortex; PCC, posterior cingulate cortex; PHC, parahippocampal cortex; PN, pontine nucleus; RN, red nucleus; RSC, retrosplenial cortex. networks (DMN-LECN, DMN-RECN, etc.) and then identified and quantified any cerebellar clusters in these intersection maps.

\section{Results}

Figure 1 displays an overview of the general cerebellar subdivisions highlighted in the various ICNs below.

\section{Sensorimotor network}

The sensorimotor network comprises the sensorimotor cortex $(\mathrm{M} 1 / \mathrm{S} 1)$, the premotor cortex (BA 6), the supplementary motor area, the anterior cingulate cortex (BA 24), the occipital cortex (BA 19/37), the temporal cortex (BA 21), and the insula (Fig. 2A). This circuit also includes the lentiform and caudate nuclei, the ventral thalami, the rostral part of the left red nucleus, and the cerebellum. Cerebellar clusters were bilaterally located within the hemispheric portion of lobules $\mathrm{V}$ and VI (Fig. 2B) and within what is likely the dorsal portion of the dentate nuclei (Fig. 2C) (although the limitations of our spatial resolution preclude a definitive distinction between the dentate nucleus and the lateral aspect of the neighboring interposed nuclei).

\section{Default mode network}

The DMN shows functional connectivity within the following cortical areas shown in Figure $3 A$ : the dorsomedial prefrontal cortex (BA 9/10), the medial prefrontal cortex (BA 32), the superior parietal cortex (BA 7), the angular gyrus (BA 39), the posterior cingulate cortex (BA 23/31), the retrosplenial cortex (BA 29/30), the medial temporal lobe, and the ventral temporal cortex (BA 20). Subcortical clusters were also found in the thalamus, the left red nucleus, and the midbrain. This diencephalo-telencephalic circuit also encompasses the cerebellum. Cerebellar clusters were bilaterally situated in the caudodorsal hemisphere of lobule IX, which may include part of the second homunculus (Fig. 3B). A small cluster was also noted in the right hemisphere of lobule VIIB. Clusters within the dorsomedian pontine nuclei were also observed (Fig. 3C).

\section{Executive network}

The right and left ECNs, RECN, and LECN, respectively, likely represent two homologous ICNs that together constitute the unitary ECN we have described previously with an ROI-based analysis (Seeley et al., 2007).

\section{LECN}

Cortical clusters were found in (Fig. 4A) the dorsolateral, mid-dorsolateral, and dorsomedial prefrontal cortex (BA 45/46, 
9, and 8), the orbitofrontal cortex (BA 47), the superior parietal cortex (BA 7), and the angular gyrus (BA 39). Subcortical clusters were also located within the left caudate nucleus. Functional connectivity was also detected in several widespread neocerebellar regions, including (Fig. $4 B$ ) the right crus I and crus II, with a crus I predominance, and limited extensions into lobules VI and VIIB, in the right rostral hemisphere of lobule IX and in the left medial crus I and crus II. Clusters were observed in the left dorsal basis pontis (Fig. 4C).

\section{RECN}

Cortical clusters were found in (Fig. 5A) the dorsolateral prefrontal cortex (BA ventral 44/45/46), the orbitofrontal cortex (BA 47), the caudal cingulate cortex (BA 23 bilaterally), the superior parietal cortex (BA 7), and the angular and supramarginal gyri (BA 39/ 40). Subcortical clusters were also located within the right caudate nucleus and the left red nucleus. Functional connectivity was also detected in several widespread neocerebellar regions, comprising (Fig. 5B) clusters located on the left side, in crus I and crus II with an extension into lobules VI and VIIB. Clusters were also present in the right dorsal basis pontis (Fig. $5 C$ ).

\section{Salience network}

The salience network demonstrates functional connectivity between (Fig. 6A) the medial frontal cortex (BA 32), the dorsal anterior cingulate cortex (BA 24), the dorsolateral prefrontal cortex (BA 46), the frontoinsular cortex (BA 47/12), the thalamus, and the red nuclei with a left predominance. Within the cerebellum (Fig. $6 B)$, clusters are located bilaterally in the lateral and ventral part of the hemisphere of lobule VI and the adjacent crus I near the posterosuperior fissure, with a narrow extension in crus II and in the hemisphere of lobule VIIB. Within lobule VI, the salience network clusters are located more laterally and closer to the posterosuperior fissure than the more paramedian clusters found in the sensorimotor network. A small region of overlap between these salience and sensorimotor clusters was present posteriorly (between $y=-62$ and $y=-67)$ on the left side. Clusters were also located in the dentate nuclei (Fig. 6C). Within the pons, clusters are situated in the region of the dorsomedian pontine nuclei.

Distinctness of the cerebellar contributions The cerebellar contributions to the five different ICNs were primarily nonoverlapping. Across all five networks shown in Figures
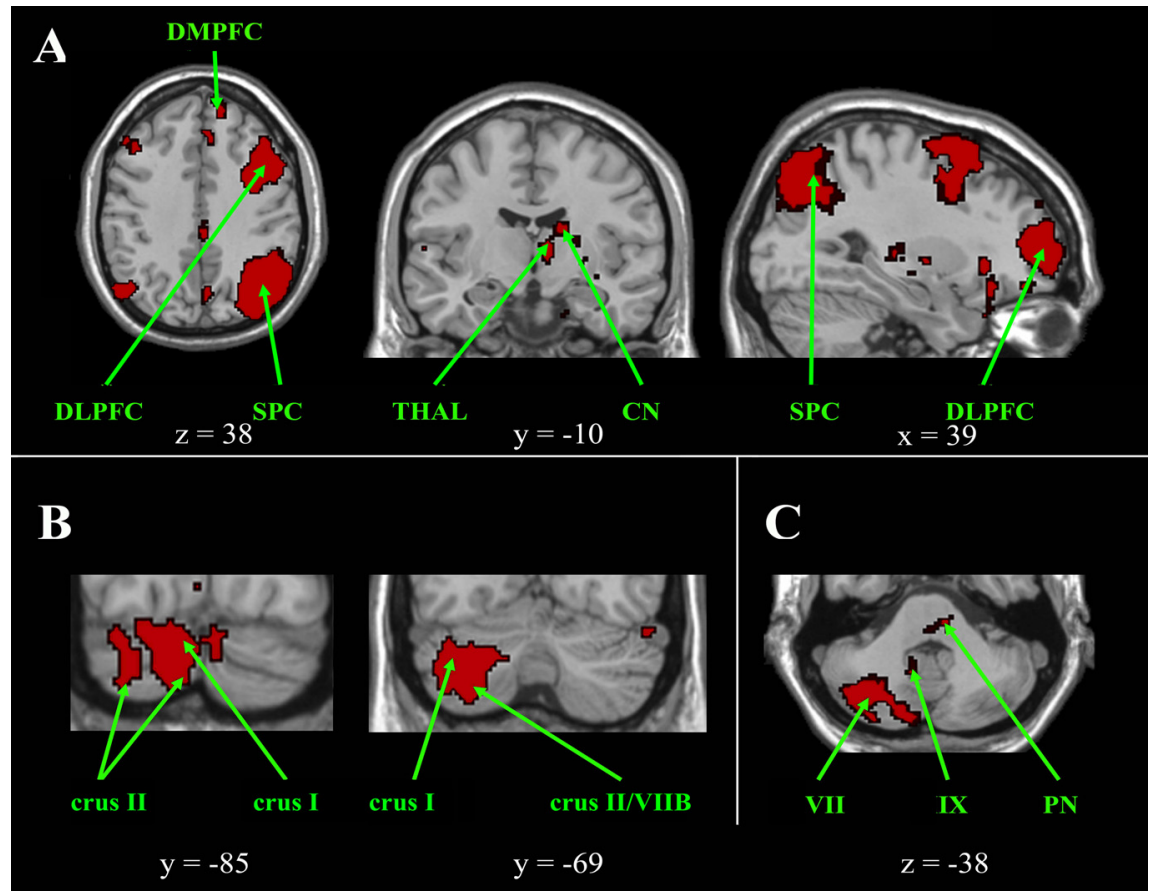

Figure 4. Cortical, subcortical, and cerebellar regions of the left executive control network. $A$, Cortical and subcortical regions of the left executive control network are shown on axial, coronal, and sagittal slices. $B, C$, Cerebellar regions are shown on coronal slices $(\boldsymbol{B})$ and on an axial slice $(\boldsymbol{C})$, which also highlights a pontine region. The left side of the image corresponds to the right side of the brain. This is an intersection map showing only voxels that were present in the left executive control network of both datasets at a corrected threshold of $p<0.01$. CN, Caudate nucleus; DLPFC, dorsolateral prefrontal cortex; DMPFC, dorsomedial prefrontal cortex; PN, pontine nucleus; SPC, superior parietal cortex; THAL, thalamus.
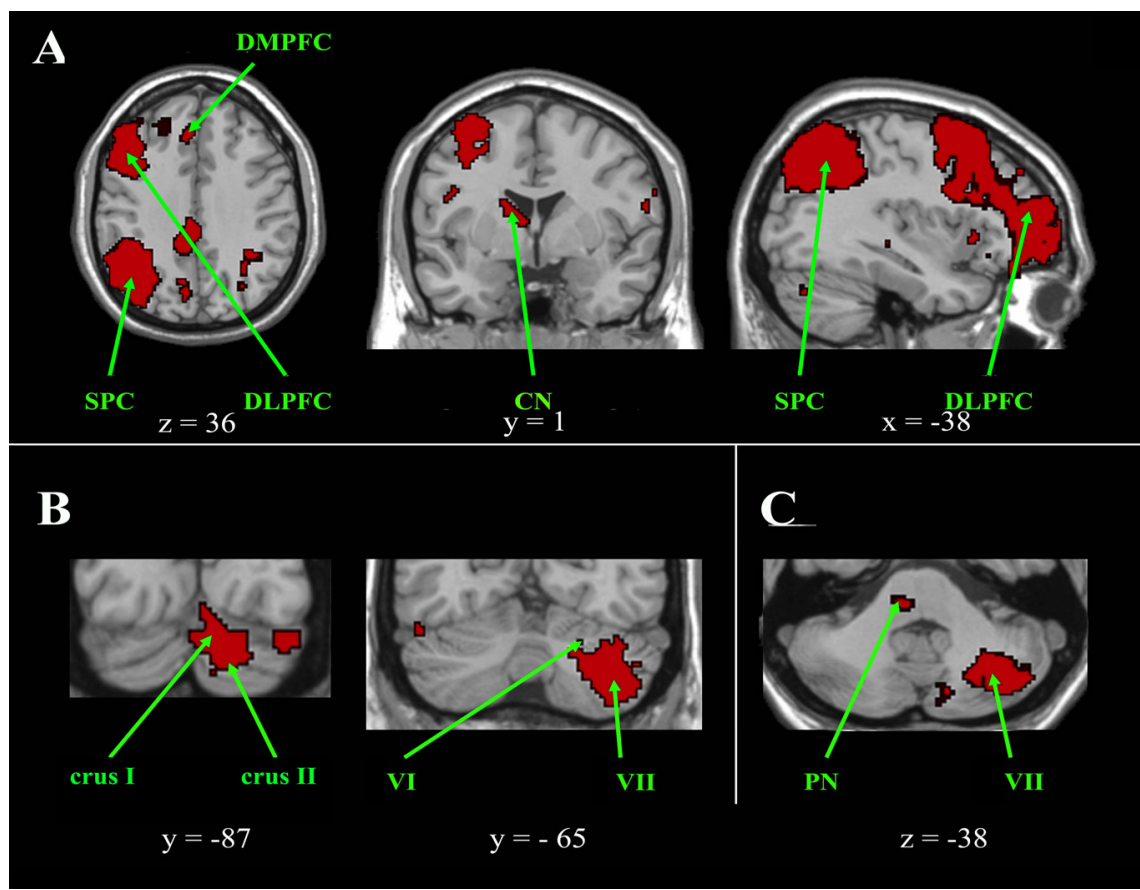

Figure 5. Cortical, subcortical, and cerebellar regions of the right executive control network. $A$, Cortical and subcortical regions of the right executive control network are shown on axial, coronal, and sagittal slices. $B, C$, Cerebellar regions are shown on coronal slices $(\boldsymbol{B})$ and on an axial slice $(\boldsymbol{C})$, which also highlights a pontine region. The left side of the image corresponds to the right side of the brain. This is an intersection map showing only voxels that were present in the right executive control network of both datasets at a corrected threshold of $p<0.01$. CN, Caudate nucleus; DLPFC, dorsolateral prefrontal cortex; DMPFC, dorsomedial prefrontal cortex; PN, pontine nucleus; SPC, superior parietal cortex. 

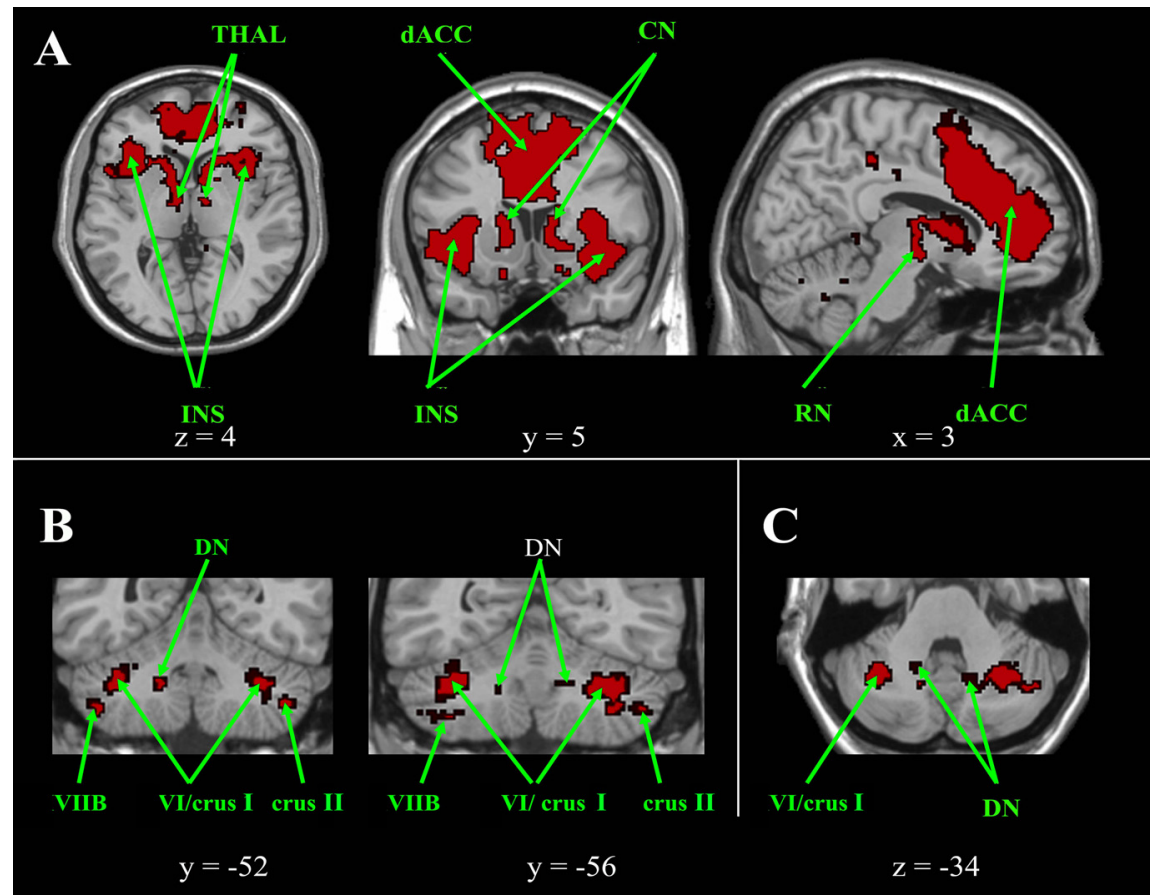

Figure 6. Cortical, subcortical, and cerebellar regions of the salience network. $\boldsymbol{A}$, Cortical and subcortical regions of the salience network are shown on axial, coronal, and sagittal slices. $\boldsymbol{B}, \boldsymbol{C}$, Cerebellar regions are shown on coronal slices $(\boldsymbol{B})$ and on an axial slice (C). The left side of the image corresponds to the right side of the brain. This is an intersection map showing only voxels that were present in the salience network of both datasets at a corrected threshold of $p<0.01$.CN, Caudate nucleus; dACC, dorsal anterior cingulate; DN, dentate nucleus; INS, insula; RN, red nucleus; THAL, thalamus.

2-6,7779 voxels were identified in the cerebellum. Of these, only 210 voxels $(<3 \%)$ appeared in more than one ICN. The cerebellar contributions to all five ICNs are shown on the same overlay in Figure 7.

\section{Discussion}

Consistent with recent task-activation studies showing replicable cerebellar responses to a variety of cognitive demands (Stoodley and Schmahmann, 2009), the current results support an expanded role of the cerebellum beyond motor control. Unlike previous ROI analyses of cerebellar connectivity (He et al., 2004; Allen et al., 2005), the current approach allows for a functional anatomic parcellation of the neocerebellum across several distinct ICNs. Our data clearly demonstrate functional coherence between the neocerebellum, particularly crus I-II, and the distinct cognitive ICNs examined here but not with the sensorimotor network. These neocerebellar networks may represent cortico-cerebellar loops as the DMN, the LECN, the RECN, and the salience network all included basis pontis clusters, presumably corresponding to the pontine nuclei, which constitute the last relay of the corticopontine fibers before their targets in the cerebellum (Schmahmann and Pandya, 1995, 1997). For the sensorimotor and salience networks, clusters were found in the dentate nuclei, which represent, with the other deep cerebellar nuclei and the lateral vestibular nucleus, the sole cerebellar output channels.

The DMN, comprised mainly of the posterior cingulate cortex/precuneus, medial prefrontal/pregenual cingulate cortices, temporoparietal regions, and medial temporal lobes, is implicated in episodic memory retrieval, self-reflection, mental imagery, and stream-of-consciousness processing (Raichle et al., 2001; Greicius et al., 2003, 2004; Buckner et al., 2005). We found that the DMN includes lobule IX and a small cluster in the right hemisphere of lobule VIIB. However, the role of lobule IX remains unclear, as in monkeys it is mainly connected to the vestibular nuclei, and its resection produces no obvious deficit (Dow and Moruzzi, 1958). The cingulate cortex projects to the pontine nuclei (Vilensky and van Hoesen, 1981), which project to the ventral paraflocculus (equivalent to lobule IX) in the cat (Brodal et al., 1991). Lobule IX has been implicated in various functional tasks including thirst satiation (Parsons et al., 2000), sensation (Hui et al., 2005), motor synchronization (Jantzen et al., 2004), working memory (Desmond et al.,1997), and perception of change in stimulus timing (Liu et al., 2008). Lobule IX, confirmed by referring to the cerebellar MRI atlas (Schmahmann et al., 2000), is involved in past and future event elaboration in conjunction with retrosplenial and precuneus cortices (Addis et al., 2007). As the most inferior portion of the cerebellum, lobule IX is often not covered by standard fMRI protocols and therefore more prone than other regions to false negatives. Nonetheless, our findings linking this region to the DMN are supported by a recent resting-state study that has also identified a DMN cluster in lobule IX (Filippini et al., 2009). The DMN, with its putative role in episodic memory retrieval and selfreflection, may be the most phylogenetically recent of the ICNs considered here and so the least likely to have obvious cerebellar homologues in nonhuman primates. The functional role of caudal lobule IX in the DMN remains unresolved. Given its replicable presence in the DMN of both datasets and in the study by Filippini et al. (2009), future studies of episodic memory, self-reflection, and other putative DMN functions should provide full coverage of the cerebellum to include this poorly understood region.

The ECN, encompassing the dorsolateral prefrontal and lateral parietal neocortices, is required for the selection and maintenance in working memory of relevant information necessary for action preparation (Seeley et al., 2007). We have shown that the ECN includes pontine clusters and the major part of the neocerebellum (crus I-II). These results agree with anatomical studies in monkeys reporting possible reciprocal connections between crus I and prefrontal cortex and crus II and parietal cortex. Specifically, afferents from the prefrontal cortex via the rostral and medial pontine nuclei converge on crus I, whereas afferents from parietal cortex converge on crus II via the lateral, dorsal, and medial pontine nuclei (Brodal, 1979). Efferents from crus I and II complete the reciprocal connection via projections from the dentate nuclei to prefrontal and parietal cortices (Dum and Strick, 2003). In humans, functional imaging studies have highlighted the role of crus I in executive functions, such as abstract reasoning (Monti et al., 2007), working memory (Chen and Desmond, 2005), information updating (Collette et al., 2007), and response selection (Desmond et al., 1998). Lesions of the neocerebellum can cause executive impairments in abstract reasoning, working memory, set-shifting, and planning (Schmahmann and Sherman, 1998). A broad functional lateralization of the cerebellum has been demonstrated, corresponding to our strongly lateralized right and left ECNs. Functional imaging (Desmond and Fiez, 


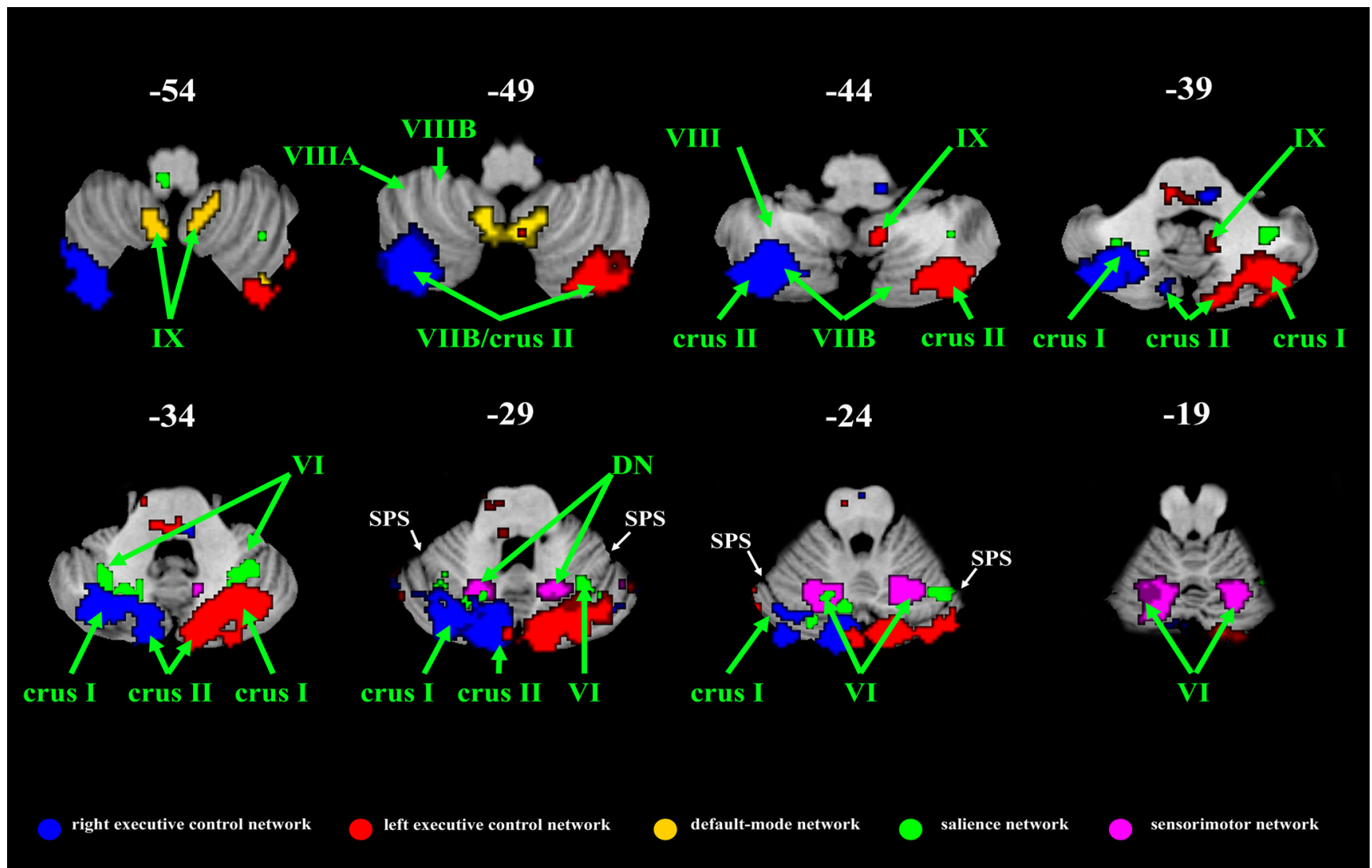

Figure 7. Distinct cerebellar contributions to the five intrinsic connectivity networks. The cerebellar clusters from all five ICN maps are overlayed on the same axial slices. With rare exceptions, such as the cluster in left lobule VI (at slices $z=-24$ and $z=-19$ ) which contributes to the sensorimotor and salience networks, there is remarkably little cerebellar overlap across the five ICNs. SPS, Superior posterior sulcus.

1998; Monti et al., 2007) and clinical studies (Gottwald et al., 2004; Richter et al., 2007) preferentially implicate the right cerebellum in verbal processes and the left cerebellum in spatial processes. Clinical and neuroimaging studies point to a consistent right neocerebellar involvement in verbal working memory and verbal fluency (Bellebaum and Daum, 2007). Impairment in verbal fluency is reported with lesions of the right crus II (Richter et al., 2007). Therefore, the neocerebellum may constitute a crucial node for verbal and nonverbal executive functions in the RECN and LECN, respectively. Finally, the right rostral lobule IX also contributes to the LECN, but as mentioned above, the function of lobule IX remains unresolved.

The salience network, centered on the dorsal anterior cingulate (BA 24/32) and frontoinsular cortices (BA 47/12) connected with subcortical limbic structures, is involved in detecting, integrating and filtering relevant interoceptive, autonomic, and emotional information (Seeley et al., 2007). The current study extends this network to two main, distinct cerebellar regions: the lateral portion of the right and left lobules VI and the adjacent crus I. Lobules VI-VII (crus I) are connected, through the pontine and dentate nuclei, with posterior and lateral hypothalamus and with the mammillary nuclei (Haines and Dietrichs, 1984; Haines et al., 1997). As the lateral neocerebellum is mainly connected to associative cortices, we postulate that the frontoinsular and prefrontal clusters detected here are preferentially linked with lobules VIcrus I. Dimitrova et al. (2003) demonstrated the role of vermal lobule VI and the hemisphere of lobules VI-crus I in pain-related processes like grimacing, fear, and startle reactions. Cerebellar strokes (hemispheres of lobules VII-VIII) cause impairment in the subjective experience of pleasant feelings (Turner et al., 2007). Frontoinsular and anterior cingulate activations have been detected during interoceptive awareness (Critchley et al., 2004) and physiological mismatch (Gray et al., 2007); notably, Gray et al. (2007) found activation in the vermis of lobule VI. We also found a small cluster overlapping the border of the vermal and paravermal lobule VI. Last, Stoodley and Schmahmann (2009) reported cerebellar activation, especially in lobule VI and crus I, during emotional processes like evaluating facial expression and empathizing. Therefore, lobule VI-crus I may contribute to estimating the valence of salient, emotional cues and selecting appropriate behavioral responses. To our surprise, we found no clusters in the posterior vermis where lesions cause affective impairments, such as blunting of emotion (Schmahmann and Sherman, 1998). A small medial/paramedial cluster was present in left lobule VI. The vermis of lobule VI receives afferents from the hypothalamus (Azizi et al., 1981) and projects back to the hypothalamus through the fastigial nuclei (Haines et al., 1997). Fastigial and interposed nucleus lesions can induce autonomic impairments (Haines et al., 1997). Stimulation of the vermis has reportedly produced improvement in some psychiatric disorders (Heath, 1977) and increased theta activity related to emotion and memory (Schutter and van Honk, 2006). Thus, vermis and paravermis of lobule VI, as detected here, could play a modulatory role on the subcortical nodes of the salience network and may represent a phylogenetically older cerebellar emotional processor in conjunction with the posterior vermis and the hemispheres of the posterior lobe. Although the lobule VI clusters in the salience and sensorimotor networks were primarily distinct, a small lob- 
ule VI cluster was found in both networks. This overlap suggests an intracerebellar connection between these two networks that merits additional study and that might relate to limbic control of the motor system.

This study has several limitations that merit consideration. Chief among them is that the networks described here are detected in the absence of specific functional activity. We are inferring functional roles for the cerebellar clusters here based on their belonging to ICNs, whose functional relevance is reasonably well established in the literature. The fact that our subjects were resting with their eyes closed does, however, avoid the critiqueleveled at standard cognitive studies of the cerebellum - that subtle motor or oculomotor requirements of the task account for the cerebellar activity (Haarmeier and Thier, 2007). The exact functional relevance of ICNs - what benefits are accrued by maintaining temporal correlations in very low-frequency neural fluctuations-remains unclear, but it seems that brain regions that typically activate together during particular tasks remain tethered at some basal level, even in the absence of their preferred task. We speculate that this basal, task-independent, intrinsic connectivity is important for avoiding disuse-related pruning of critical synapses (Luo and O'Leary, 2005). Others have suggested that this basal connectivity maintains networks in a primed state to improve response efficiency (Fox and Raichle, 2007). We were encouraged by the sensitivity of the approach used here to detect, in several ICNs, clusters in the pontine and dentate nuclei, the major convergence points for afferent and efferent connections, respectively. It should be noted, however, that both the pontine and dentate nuclei should be present in each of the five ICNs studied here (Schmahmann, 2002). Our insistence on replicating clusters across both datasets may have been too stringent to allow for the detection of clusters in these smaller nuclei in all five ICNs. The same can be said for the unilateral contribution of the (left) red nucleus to the sensorimotor cortex where we would expect bilateral involvement of the red nuclei in this ICN. The spatial resolution of the current study does not allow us to definitively distinguish the medial aspect of the dentate nucleus and the lateral aspect (emboliform nucleus) of the adjacent interposed nucleus. Finally, although the convergence of our results with more precise tract-tracing studies in animals is reassuring, it is important to note that the BOLD signal used in fMRI is only an indirect measure of neural activity.

In conclusion, the present study provides further support for the view that distinct neocerebellar regions are involved in distinct cognitive functions. The human neocerebellum, particularly lobules VI and VII (crus I-II), selectively contributes to parallel cortico-cerebellar loops involved in executive control, salience detection, and episodic memory/self-reflection. The greatest portions of the neocerebellum contributed to the ECN, a network involved with selection and maintenance in working memory of relevant multimodal information. Interestingly, lobule IX, whose functional significance remains unresolved, was a major component of the DMN and, to a lesser degree, the LECN. These findings should help guide subsequent investigations designed to specify the precise functional role of distinct cerebellar regions in higher order cognitive and affective processing.

\section{References}

Addis DR, Wong AT, Schacter DL (2007) Remembering the past and imagining the future: common and distinct neural substrates during event construction and elaboration. Neuropsychologia 45:1363-1377.

Allen G, McColl R, Barnard H, Ringe WK, Fleckenstein J, Cullum CM (2005) Magnetic resonance imaging of cerebellar-prefrontal and cerebellarparietal functional connectivity. Neuroimage 28:39-48.
Azizi SA, Mihailoff GA, Burne RA, Woodward DJ (1981) The pontocerebellar system in the rat: an HRP study. I. Posterior vermis. J Comp Neurol 197:543-548.

Beckmann CF, Smith SM (2004) Tensorial extension of independent component analysis for multi-subject FMRI analysis. IEEE Trans Med Imaging 23:137-152.

Beckmann CF, DeLuca M, Devlin JT, Smith SM (2005) Investigations into resting-state connectivity using independent component analysis. Philos Trans R Soc Lond B Biol Sci 360:1001-1013.

Bellebaum C, Daum I (2007) Cerebellar involvement in executive control. Cerebellum 6:184-192.

Biswal B, Yetkin FZ, Haughton VM, Hyde JS (1995) Functional connectivity in the motor cortex of resting human brain using echo-planar MRI. Magn Reson Med 34:537-541.

Brodal P (1979) The pontocerebellar projections in the rhesus monkey: an experimental study with retrograde axonal transport of horseradish peroxydase. Neuroscience 4:193-208.

Brodal P, Bjaalie JG, Aas JE (1991) Organization of the cingulo-pontocerebellar connections in the cat. Anat Embryol (Berlin) 184:245-254.

Buckner RL, Snyder AZ, Shannon BJ, LaRossa G, Sachs R, Fotenos AF, Sheline YI, Klunk WE, Mathis CA, Morris JC, Mintun MA (2005) Molecular, structural, and functional characterization of Alzheimer's disease: evidence for a relationship between default activity, amyloid, and memory. J Neurosci 25:7709-7717.

Chen SH, Desmond JE (2005) Cerebrocerebellar networks during articulatory rehearsal and verbal memory tasks. Neuroimage 24:332-338.

Collette F, Van der Linden M, Laureys S, Arigoni F, Delfiore G, Degueldre C, Luxen A, Salmon E (2007) Mapping the updating process: common and specific brain activations across different versions of the running span task. Cortex 43:146-158.

Cordes D, Haughton VM, Arfanakis K, Wendt GJ, Turski PA, Moritz CH, Quigley MA, Meyerand ME (2000) Mapping functionally related regions of brain with functional connectivity MR imaging. AJNR Am J Neuroradiol 21:1636-1644.

Critchley HD, Wiens S, Rotshtein P, Ohman A, Dolan RJ (2004) Neural systems supporting interoceptive awareness. Nat Neurosci 7:189-195.

Damoiseaux JS, Rombouts SA, Barkhof F, Scheltens P, Stam CJ, Smith SM, Beckmann CF (2006) Consistent resting-state networks across healthy subjects. Proc Natl Acad Sci U S A 103:13848-13853.

Desmond JE, Fiez JA (1998) Neuroimaging studies of the cerebellum: language, learning, memory. Trends Neurosci 2:355-362.

Desmond JE, Gabrieli JD, Wagner AD, Ginier BL, Glover GH (1997) Lobular patterns of cerebellar activation in verbal working-memory and finger-tapping tasks as revealed by functional MRI. J Neurosci 17:9675-9685.

Desmond JE, Gabrieli JD, Glover GH (1998) Dissociation of frontal and cerebellar activity in a cognitive task: evidence for a distinction between selection and search. Neuroimage 7:368-376.

Dimitrova A, Kolb FP, Elles HG, Maschke M, Forsting M, Diener HC, Timmann D (2003) Cerebellar responses evoked by nociceptive leg withdrawal reflex as revealed by event-related FMRI. J Neurophysiol 90:1877-1886.

Dow RS, Moruzzi G (1958) The physiology and pathology of the cerebellum. Minneapolis: University of Minnesota.

Dum RP, Strick PL (2003) An unfolded map of the cerebellar dentate nucleus and its projection to the cerebral cortex. J Neurophysiol 89:634-639.

Filippini N, MacIntosh BJ, Hough MG, Goodwin GM, Frisoni GB, Smith SM, Matthews PM, Beckmann CF, Mackay CE (2009) Distinct patterns of brain activity in young carriers of the APOE-epsilon4 allele. Proc Natl Acad Sci U S A 106:7209-7214.

Fox MD, Raichle ME (2007) Spontaneous fluctuations in brain activity observed with functional magnetic resonance imaging. Nat Rev Neurosci $8: 700-711$.

Fox MD, Snyder AZ, Vincent JL, Corbetta M, Van Essen DC, Raichle ME (2005) The human brain is intrinsically organized into dynamic, anticorrelated functional networks. Proc Natl Acad Sci U S A 102:9673-9678.

Fransson P (2005) Spontaneous low-frequency BOLD signal fluctuations: an $\mathrm{fMRI}$ investigation of the resting-state default mode of brain function hypothesis. Hum Brain Mapp 26:15-29.

Glover GH, Lai S (1998) Self-navigated spiral fMRI: interleaved versus single-shot. Magn Reson Med 39:361-368. 
Gottwald B, Wilde B, Mihajlovic Z, Mehdorn HM (2004) Evidence for distinct cognitive deficits after focal cerebellar lesions. J Neurol Neurosurg Psychiatry 75:1524-1531.

Gray MA, Harrison NA, Wiens S, Critchley HD (2007) Modulation of emotional appraisal by physiological feedback during fMRI. PloS ONE 2:e546.

Greicius MD, Krasnow B, Reiss AL, Menon V (2003) Functional connectivity in the resting brain: a network analysis of the default mode hypothesis. Proc Natl Acad Sci U S A 100:253-258.

Greicius MD, Srivastava G, Reiss AL, Menon V (2004) Default-mode network activity distinguishes Alzheimer's disease from healthy aging: evidence from functional MRI. Proc Natl Acad Sci U S A 101:4637-4642.

Greicius MD, Flores BH, Menon V, Glover GH, Solvason HB, Kenna H, Reiss AL, Schatzberg AF (2007) Resting-state functional connectivity in major depression: abnormally increased contributions from subgenual cingulate cortex and thalamus. Biol Psychiat 62:429-437.

Greicius MD, Supekar K, Menon V, Dougherty RF (2009) Resting-state functional connectivity reflects structural connectivity in the default mode network. Cereb Cortex 19:72-78.

Haarmeier T, Thier P (2007) The attentive cerebellum-myth or reality? Cerebellum 6:177-183.

Haines DE, Dietrichs E (1984) An HRP study of hypothalamocerebellar and cerebellohypothalamic connections in squirrel monkey (Saimiri sciureus). J Comp Neurol 229:559-575.

Haines DE, Dietrichs E, Mihailoff GA, McDonald EF (1997) Cerebellarhypothalamic axis: basis circuits and clinical observations. Int Rev Neurobiol 41:83-107.

He Y, Zang Y, Jiang T, Liang M, Gong G (2004) Detecting functional connectivity of the cerebellum using low frequency fluctuations (LFFs). LNCS 3217:907-915.

Heath RG (1977) Modulation of emotion with a brain pacemaker. J Nerv Ment Dis 165:300-317.

Helmuth LL, Ivry RB, Shimizu N (1997) Preserved performance by cerebellar patients on tests of word generation, discrimination learning and attention. Learn Mem 3:456-474.

Hui KK, Liu J, Marina O, Napadow V, Haselgrove C, Kwong KK, Kennedy DN, Makris N (2005) The integrated response of the human cerebrocerebellar and limbic systems to acupuncture stimulation at ST 36 as evidenced by fMRI. Neuroimage 27:479-496.

Jantzen KJ, Steinberg FL, Kelso JA (2004) Brain networks underlying human timing behavior are influenced by prior context. Proc Natl Acad Sci U S A 101:6815-6820.

Kelly RM, Strick PL (2003) Cerebellar loops with motor cortex and prefrontal cortex of a nonhuman primate. J Neurosci 23:8432-8444.

Kim DH, Adalsteinsson E, Glover GH, Spielman DM (2002) Regularized higher-order in vivo shimming. Magn Reson Med 48:715-722.

Kiviniemi V, Kantola JH, Jauhiainen J, Hyvärinen A, Tervonen O (2003) Independent component analysis of nondeterministic fMRI signal sources. Neuroimage 19:253-260.

Laufs H, Krakow K, Sterzer P, Eger E, Beyerle A, Salek-Haddadi A, Kleinschmidt A (2003) Electroencephalographic signatures of attentional and cognitive default modes in spontaneous brain activity fluctuations at rest. Proc Natl Acad Sci U S A 100:11053-11058.

Levisohn L, Cronin-Golomb A, Schmahmann JD (2000) Neuropsychological consequences of cerebellar tumour resection in children. Cerebellar cognitive affective syndrome in paediatric population. Brain 123:1041-1050.

Liu T, Xu D, Ashe J, Bushara K (2008) The specificity of inferior olive response to stimulus timing. J Neurophysiol 100:1557-1561.

Luo L, O'Leary DD (2005) Axon retraction and degeneration in development and disease. Annu Rev Neurosci 28:127-156.

Middleton FA, Strick PL (1997) Dendate output channels: motor and cognitive components. Prog Brain Res 114:553-566.

Middleton FA, Strick PL (2001) Cerebellar projections to the prefrontal cortex of the primate. J Neurosci 21:700-712.
Monti MM, Osherson DN, Martinez MJ, Parsons LM (2007) Functional neuroanatomy of deductive inference: a language-independent distributed network. Neuroimage 37:1005-1016.

Parsons LM, Denton D, Egan G, McKinley M, Shade R, Lancaster J, Fox PT (2000) Neuroimaging evidence implicating cerebellum in support of sensory/cognitive processes associated with thirst. Proc Natl Acad Sci U S A 97:2332-2336.

Poline JB, Worsley KJ, Evans AC, Friston KJ (1997) Combining spatial extent and peakintensity to test for activations in functional imaging. Neuroimage 5:83-96.

Raichle ME, MacLeod AM, Snyder AZ, Powers WJ, Gusnard DA, Shulman GL (2001) A default mode of brain function. Proc Natl Acad Sci U S A 98:676-682.

Ramnani N, Behrens TE, Johansen-Berg H, Richter MC, Pinsk MA, Andersson JL, Rudebeck P, Ciccarelli O, Richter W, Thompson AJ, Gross CG, Robson MD, Kastner S, Matthews PM (2006) The evolution of prefrontal inputs to the cortico-pontine system: diffusion imaging evidence from macaque monkeys and humans. Cereb Cortex 16:811-818.

Richter S, Gerwig M, Aslan B, Wilhelm H, Schoch B, Dimitrova A, Gizewski ER, Ziegler W, Karnath HO, Timmann D (2007) Cognitive functions in patients with MR-defined chronic focal cerebellar lesions. J Neurol 254:1193-1203.

Schmahmann JD (1996) From movement to thought: anatomic substrates of the cerebellar contribution to cognitive processing. Hum Brain Mapp 4:174-198.

Schmahmann JD (2002) The role of the cerebellum in affect and psychosis. In: The cerebellum and its disorders (Manto MU, Pandolfo M, eds), pp 136-160. Cambridge, UK: Cambridge UP.

Schmahmann JD (2004) Disorders of the cerebellum: ataxia, dysmetria of thought, and the cerebellar cognitive affective syndrome. J Neuropsychiatry Clin Neurosci 16:367-378.

Schmahmann JD, Pandya DN (1995) Prefrontal cortex projections to the basilar pons: implications for the cerebellar contribution to higher function. Neurosci Lett 199:175-178.

Schmahmann JD, Pandya DN (1997) The cerebrocerebellar system. Int Rev Neurobiol 41:31-60.

Schmahmann JD, Sherman JC (1998) The cerebellar cognitive and affective syndrome. Brain 121:561-579.

Schmahmann JD, Doyon J, Toga AW, Petrides M, Evans AC (2000) MRI atlas of the human cerebellum. San Diego: Academic.

Schutter DJ, van Honk J (2006) An electrophysiological link between the cerebellum cognition and emotion: frontal theta EEG activity to singlepulse cerebellar TMS. Neuroimage 33:1227-1231.

Seeley WW, Menon V, Schatzberg AF, Keller J, Glover GH, Kenna H, Reiss AL, Greicius MD (2007) Dissociable intrinsic connectivity networks for salience processing and executive control. J Neurosci 27:2349-2356.

Stoodley CJ, Schmahmann JD (2009) Functional topography in the human cerebellum: a meta-analysis of neuroimaging studies. Neuroimage 44:489-501.

Thier P, Haarmeier T, Treue S, Barash S (1999) Absence of a common functional denominator of visual disturbances in cerebellar disease. Brain 122:2133-2146.

Turner BM, Paradiso S, Marvel CL, Pierson R, Boles Ponto LL, Hichwa RD, Robinson RG (2007) The cerebellum and emotional experience. Neuropsychologia 45:1331-1341.

Vilensky JA, van Hoesen GW (1981) Corticopontine projections from the cingulate cortex in the rhesus monkey. Brain Res 205:391-395.

Vincent JL, Patel GH, Fox MD, Snyder AZ, Baker JT, Van Essen DC, Zempel JM, Snyder LH, Corbetta M, Raichle ME (2007) Intrinsic functional architecture in the anaesthetized mokey brain. Nature 447:83-86.

Xiong J, Parsons LM, Gao JH, Fox PT (1999) Interregional connectivity to primary motor cortex revealed using MRI resting state images. Hum Brain Mapp 8:151-156. 